\title{
Novel blue continuous-wave current-injected light source
}

Tien-Chang Lu, Shing-Chung Wang, Shih-Wei Chen, and Hao-Chung Kuo

An electrically pumped gallium nitride vertical-cavity surfaceemitting laser exhibits promising performance at a wavelength of $462 \mathrm{~nm}$, achieved with excellent electrical properties.

Vertical-cavity surface-emitting lasers (VCSELs) contain two high-reflectivity distributed-Bragg reflectors (DBRs) as well as a short cavity characterized by a small optical-mode volume. They emit a single longitudinal mode in a circularly symmetric beam with little beam divergence. Both characteristics are superior to those achieved with edge-emitting lasers and desirable for many practical applications in, for example, high-density optical storage and laser printing. In addition, the strong field in the microcavity facilitates exploration of cavity quantum-electrodynamics effects, such as single-photon and polariton emission, controlled spontaneous emission, and low threshold (or thresholdless) lasing.

Over the past decade, two developments have affected the realization of electrically pumped gallium nitride $(\mathrm{GaN})$-based VCSELs. First, high-reflectivity, high-quality DBRs composed of $\mathrm{Al}_{x} \mathrm{Ga}_{1-x} \mathrm{~N}$ and $\mathrm{GaN}$ exhibit a large lattice mismatch that forms cracks in the epitaxially grown DBR structure. These cracks could result in a reduction in optical reflectivity and become a leakage-current path. The second is the fabrication of novel, high-transparency, high-conductivity contacts for current injection, which was triggered by the difficulties encountered in constructing low-resistance p-type GaN layers.

In 1996, GaN-based edge-emitting laser diodes were first realized at room temperature by Nakamura's team. ${ }^{1}$ Arakawa and co-workers subsequently fabricated an $\operatorname{In}_{0.1} \mathrm{Ga}_{0.9} \mathrm{~N}$ VCSEL and observed lasing at 77K for the first time in $1998 .^{2}$ We recently fabricated a crack-free high-reflectivity DBR by incorporating AlN/GaN superlattice layers between the DBR structures to reduce $\mathrm{V}$-shaped defects and act as strain buffers. ${ }^{3}$

Figure 1 shows the device structure employing hybrid mirrors. It consists of 29 pairs of AlN/GaN DBRs and a $5 \lambda$ (where $\lambda$ refers to the operating wavelength) active region with a

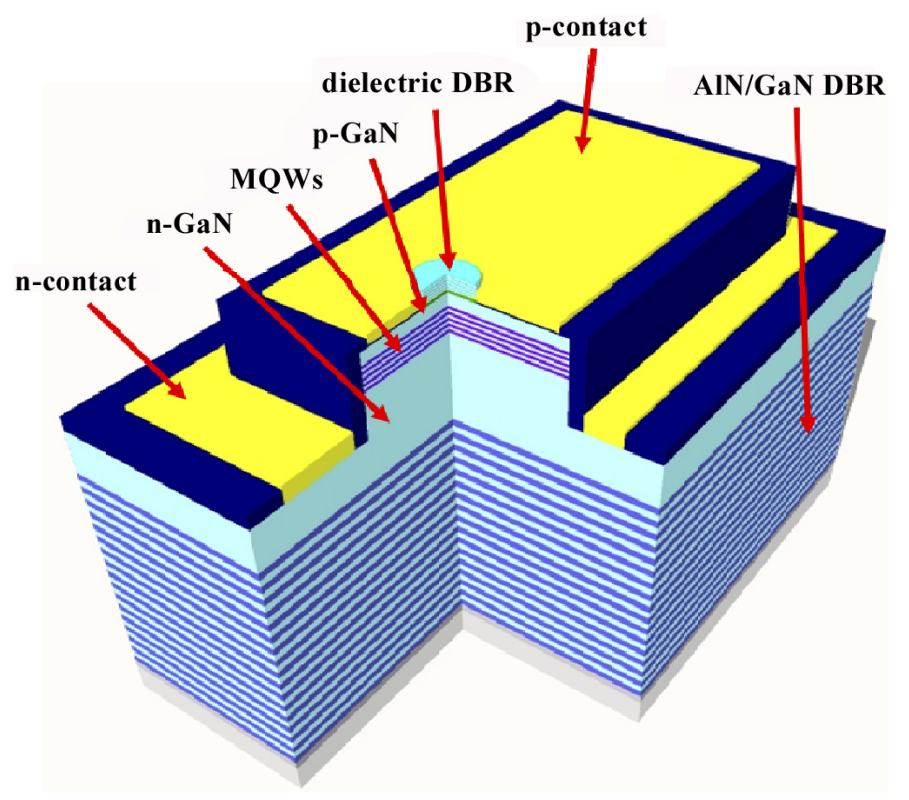

Figure 1. Schematic diagram of an electrically pumped vertical-cavity surface-emitting laser. DBR: Distributed-Bragg reflector. GaN, AlN: Gallium, aluminum nitride. MQW: Multiquantum well.

790nm-thick n-type GaN layer, 10 pairs of InGaN/GaN mutiple quantum wells, and a 120nm-thick p-type GaN layer. The DBR showed a high peak reflectivity of $99.4 \%(\lambda=450 \mathrm{~nm})$. A $0.2 \mu \mathrm{m}-$ thick $\mathrm{SiN}_{x}$ layer was used as current block to limit the currentinjection area to $10 \mu \mathrm{m}$ in diameter. We used indium tin oxide (ITO) as our transparent contact layer on top of the p-type GaN film to reduce device resistance. ITO offers excellent properties (such as high transmission and great conductivity) to realize the first continuous-wave (CW) current-injected blue GaN VCSEL. We also deposited $\mathrm{p}$ and $\mathrm{n}$ contacts as current-injection layers. Finally, an eight-pair $\mathrm{Ta}_{2} \mathrm{O}_{5} / \mathrm{SiO}_{2}$ dielectric DBR (with a measured reflectivity of about $99 \%$ at $\lambda=450 \mathrm{~nm}$ ) was deposited as the top DBR mirror to complete the full hybrid microcavityVCSEL device. ${ }^{4}$ 


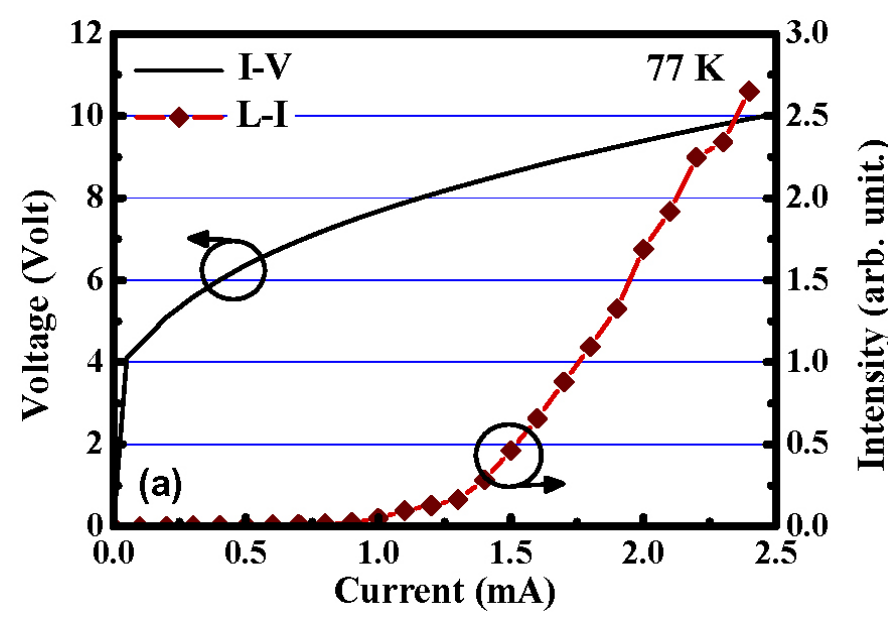

Figure 2. Injection current as a function of voltage and emission intensity. I-V: Current-voltage (left-hand y axis). L-I: Current-intensity (right-hand y axis).

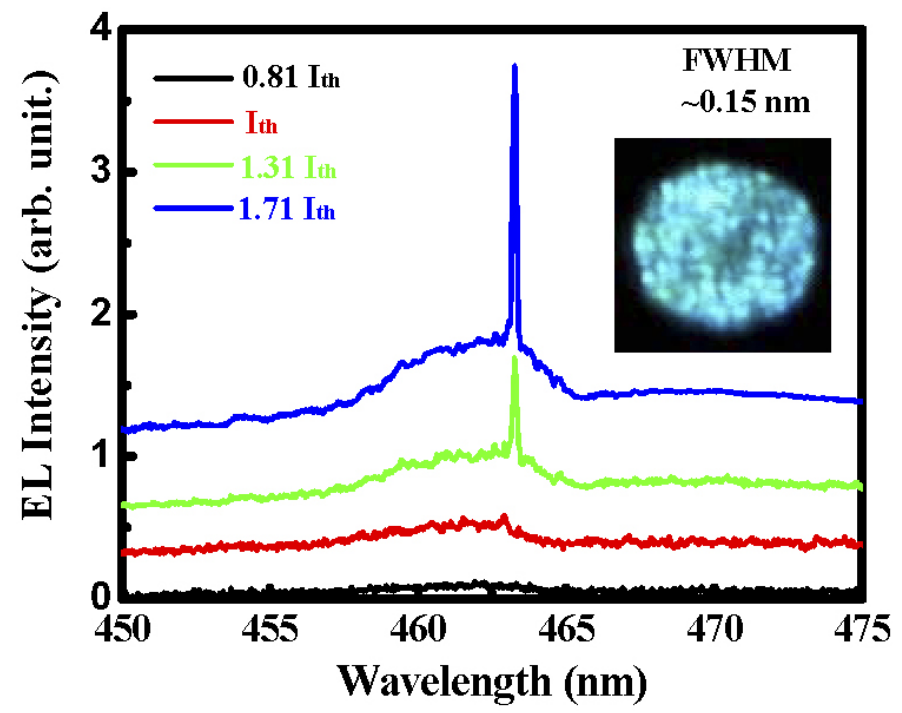

Figure 3. Electroluminescence (EL) spectrum for different injection currents. FWHM: Full width at half maximum. Ith: Threshold current.

We measured the VCSELs under CW current injection at 77K. Figure 2 shows the light output intensity as a function of injection current and current-voltage characteristics of the VCSEL sample. The turn-on voltage was about $4.1 \mathrm{~V}$, thus showing the laser's good electrical properties. The laser was characterized by a clear threshold current at $1.4 \mathrm{~mA}$, which we increased linearly beyond the threshold.

Figure 3 shows the electroluminscence spectrum for various injection currents (above the threshold) and a dominant laser wavelength of $462.8 \mathrm{~nm}$. The inset image shows the $10 \mu \mathrm{m}$ emission aperture at $1 \mathrm{~mA}$, where we observe the nonuniform emission intensity in the form of several bright emission spots (caused by indium fluctuations in the multiquantum wells).

In conclusion, we fabricated and demonstrated an electrically pumped GaN-based VCSEL at 77K. The crack-free, highreflectivity DBR and high-quality ITO layer facilitated lasing at a threshold current of $1.4 \mathrm{~mA}$ for a dominant wavelength of $462.8 \mathrm{~nm}$ at $77 \mathrm{~K}$. The turn-on voltage was $4.1 \mathrm{~V}$, with great electrical characteristics. As our next steps we still need to resolve a few technical issues, such as improvement of the epitaxial quality, better current and optical confinement, and advanced structure design, before a CW GaN-based VCSEL can be realized at room temperature.

This work was supported in part by the National Science Council of Taiwan under contract NSC 96-2221-E009-094-MY3.

\section{Author Information}

Tien-Chang Lu, Shing-Chung Wang, Shih-Wei Chen, and Hao-Chung Kuo

Department of Photonics

National Chiao-Tung University

Hsin-chu, Taiwan

Tien-Chang Lu received his $\mathrm{PhD}$ in electrical engineering and computer science from the National Chiao-Tung University in 2004. Since 2005, he has been a member of faculty. His research work includes VCSELs, photonic-crystal surface-emitting lasers (PCSELs), and microcavities.

Shing-Chung Wang received his PhD in electrical engineering from Stanford University in 1971. Since 1995, he has been a faculty member at the Institute of Electro-Optical Engineering. His current research interests include semiconductor nanostructures and VCSELs.

Shih-Wei Chen is a PhD student in electro-optical engineering. In 2006, he joined the Semiconductor Laser Technology Laboratory. His research interests are in the areas of LEDs, GaN VCSELs, high-Q (quality) microcavity LEDs, and PCSELs.

Hao-Chung Kuo received his $\mathrm{PhD}$ in electrical and computer engineering from the University of Illinois at Urbana-Champaign in 1999. Since 2002, he has been a faculty member at the 
Institute of Electro-Optical Engineering. His research work includes LEDs, laser disks, and solar cells.

\section{References}

1. S. Nakamura, M. Senoh, S.-I. Nagahama, N. Iwasa, T. Yamada, T. Matsushita, Y. Sugimoto, and H. Kiyoku, Room-temperature continuous-wave operation of In GaN multi-quantum-well structure laser diodes, Appl. Phys. Lett. 69 (26), pp. 4056-4058, 1996.

2. T. Someya, K. Tachibana, J. Lee, T. Kamiya, and Y. Arakawa, Lasing emission from an $\mathrm{In}_{0.1} \mathrm{G} a_{0.9} \mathrm{~N}$ vertical cavity surface emitting laser, Jpn. J. Appl. Phys. 37, pp. L1424L1426, 1998.

3. G. S. Huang, T. C. Lu, H. H. Yao, H. C. Kuo, S. C. Wang, C.-W. Lin, and L. Chang, Crack-free GaN/AlN distributed Bragg reflectors incorporated with GaN/AlN superlattices grown by metalorganic chemical vapor deposition, Appl. Phys. Lett. 88 (6), p. 061904, 2006.

4. T. C. Lu, C. C. Kao, H. C. Kuo, G. S. Huang, and S. C. Wang, CW lasing of current injection blue GaN-based vertical cavity surface emitting laser, Appl. Phys. Lett. 92 (14), p. 141102, 2008. 